\title{
El uso del estudio de casos en la investigación en psicoterapia
}

\author{
Mariano G. Scandar ${ }^{1}$
}

\section{Artículo}

Material original autorizado para la publicación en la revista Psicodebate. Facultad de Ciencias Sociales. Universidad de Palermo.

Recibido 21-02-2014 | Aceptado 23-04-2014

\section{Resumen}

Este trabajo revisa el estado actual de la investigación mediante Estudio de Casos (EC) en psicoterapia. El uso de esta metodología se remonta a los comienzos de la disciplina y es hoy una forma de investigación utilizada ampliamente. Sin embargo, frecuentemente se ha criticado su falta de rigurosidad y sus dificultades para extraer de ella conocimientos generalizables. Existe un cierto nivel de vaguedad en la forma en que es utilizado el término "Estudio de Caso" en la bibliografía. En este trabajo, primeramente se define un EC como una investigación en profundidad de un caso en su contexto, buscando ver sus particularidades y determinaciones dadas por su anclaje temporo-espacial. En segundo lugar, se enumeran diversos tipos de EC utilizados en psicoterapia así como las guías que diversos autores han sugerido para la realización sistemática y confiable de este tipo de trabajos. Se da cuenta de las ventajas de los EC y de las críticas más frecuentes. Se concluye en la importancia de sistematizar la forma en que deben realizarse este tipo de investigaciones a las que se considera una herramienta indispensable para comprender los procesos terapéuticos y para probar la plausibilidad de las hipótesis y proposiciones teóricas.

Palabras Clave: Investigación en Psicoterapia - Estudios de Caso - Métodos Cualitativos - Métodos Mixtos.

1 Fundación de Neuropsicología Clínica; Fundación ETCl, mariano@fnc.org.ar 


\section{Abstract}

This article reviews the current state of the use of Case Studies (CS) in psychotherapy research. This technique has been used since the beginnings of the discipline and is still a widely used form of research. However, it has been often criticized because of its lack of rigor and the difficulties to extract generalizable knowledge from individual cases. There is a certain level of vagueness in the way "case study" is used the term in the literature. In this paper, first we define a CS as an in-depth investigation of a case in its context, seeking to see their particularities and boundaries. Secondly we list various types of CS used in psychotherapy and the guidelines that several authors have suggested to guarantee reliability in CS. We summarize the benefits and weakness of the CS research. We conclude the importance of systematizing the way CS should be done. This type of research is an essential tool for understanding the therapeutic process and for testing the plausibility of hypotheses and theoretical propositions.

Keywords: Psychotherapy Research - Case Study - Qualitative methods Mixed Methods. 
La investigación mediante Estudios de Caso (EC) puede rastrearse históricamente a los inicios de la psicoterapia. Stiles (2005) cita los célebres historiales freudianos (Dora, El Hombre de los Lobos, etc.) y el paradigmático caso del Pequeño Albert, de Watson, como ejemplos de EC fundacionales. En ese mismo sentido, y refiriéndose a la terapia familiar, Dattilio, recuerda que "en los días iniciales de la terapia familiar, no se distinguía entre la terapia y la investigación" (Dattilio, 2006, p. 208), destacando cómo todo el conocimiento teórico era extraído de la praxis, que se cristalizaba en la presentación de EC para ser comunicados.

Paulatinamente, sobre todo en los últimos 25 años, los tratamientos basados en la evidencia cobran fuerza en el campo de la psicoterapia y se comienza a exigir a todo abordaje que pruebe empíricamente su eficacia (Edwards, Dattilio, \& Bromley, 2004). Este afán de validación resulta un avance tanto para el desarrollo de la psicoterapia como para sus usuarios. Sin embargo, ha tenido por resultado indeseado que se equipare erróneamente "tratamiento empíricamente validado" con "estudio controlado randomizado (ECR)".

Los ECR tienen su origen en el campo de la medicina y consisten en la asignación aleatoria de sujetos a diferentes opciones de tratamiento sin que ni el experimentador ni el sujeto estén al tanto de qué opción terapéutica se está aplicando (doble ciego). En el campo de la psicoterapia tanto el uso del azar como la operacionalización de variables en términos cuantitativos resulta compleja y en algunos casos imposible. Sin embargo, los ECR se utilizan con éxito en la validación de protocolos de tratamiento y son considerados actualmente el estándar de máxima calidad en investigación (Kazdin \& Blase, 2011), desplazando parcialmente otro tipo de metodologías, también de base empírica, como lo es el EC (Stiles, 2005).

Eduards y colaboradores (2004) señalan como causas de esta progresiva expansión del modelo médico al campo de las psicoterapias, razones vinculadas no tanto con variantes epistemológicas sino con razones de mercado: por un lado, los prestadores de salud mental buscan diagnósticos precisos que permitan determinar el monto mínimo de psicoterapia que es necesaria creando una presión sobre los tratamientos basados en la evidencia para que sean a un tiempo estandarizados y breves. Paralelamente, como los prestadores se basan en el modelo médico, existe una presión para que los tratamientos sean evaluados del mismo modo que los tratamientos farmacológicos, es decir, ECR.

Otro aspecto a considerar es que el uso del término "Estudio de Caso" se ha vuelto ambiguo, agrupando investigaciones que difieren entre sí tanto en sus objetivos como en sus métodos (Iwakabe \& Gazzola, 2009). Esto dificulta la valoración del aporte real de los EC al conocimiento de la praxis psicoterapéutica. 
La intención de este trabajo es: definir el EC, dar cuenta de su utilidad en el campo de la psicoterapia, destacar los avances metodológicos en los diseños los EC y evaluar sus fortalezas y debilidades.

\section{Hacia una definición de los estudios de casos}

Tight inicia sus reflexiones sobre los EC señalando que "cualquiera que haga una búsqueda bibliográfica con el término EC encontrará cientos sino miles de artículos y libros aparentemente relevantes, la mayoría de los cuales, sin embargo no se relacionan con la presente discusión" (Tight, 2010, p. 329). Esta experiencia es fácilmente replicable y nos confronta con la necesidad de arribar a una definición más precisa que nos permita luego evaluar sus beneficios y debilidades.

Existe según Kyburz-Graber (2004) una tentación por parte de muchos de los investigadores noveles en ciencias sociales de realizar pretendidos EC como una forma sencilla, rápida y poco trabajosa de dar a conocer sus experiencias y acceder a publicaciones. En contraposición, numerosos autores han intentado reducir y consensuar qué diseños de investigación pueden considerase dentro de este campo buscando dotar a los EC de un estatus científico aceptable sin que este camino los obligue a resignar su esencia fenomenológica y convertirse en estudios cuantitativos intrasujeto (Kyburz-Graber, 2004).

Puede encontrarse un relativo consenso entre los autores, respecto a que un EC implica "La examinación o investigación de forma intensiva, detallada y profunda de una unidad simple: el caso" (Luck, Javkdon, \& Usher, 2006, p. 104). El "caso" es un fenómeno puntual que puede definirse con coordenadas espaciales y temporales. Por lo tanto, se encuentra ligado a su contexto de forma irreductible, al punto que su análisis descontextualizado carece de sentido. Cuanto más difusos son los límites entre el caso y su contexto, más pertinente se vuelve el uso de EC. Su fortaleza está justamente en poder incluir de forma deliberada condiciones contextuales de forma holística sin intentar individualizar ni controlar variables (Yin, 2003).

Preguntarse qué es un EC, en el contexto antes descripto, está lejos de ser una pregunta capciosa. La literatura suele considerarlo de forma diversa: como un método, como una herramienta, como un tipo de investigación cualitativa o como una estrategia de investigación (Staker, 2000; Starman, 2013; Stevenson, 2004; Yin, 2003).

Para Starman (2013), por ejemplo, el EC debe considerarse como un tipo de investigación cualitativa aun cuando incluya datos cuantitativos, ya que al elegir este tipo de investigación, estamos inevitablemente en el campo de la interpretación fenomenológica de los datos y nos alejamos de la búsqueda de la generalización estadística. Autores de corrientes metodológicas diversas acuerdan en considerar que los objetivos que guían a un EC son siempre la profundización 
y la comprensión de un fenómeno (Luck, Javkdon, \& Usher, 2006). Por oposición, entonces, ni la narración anecdótica de una experiencia terapéutica, ni los estudios estadísticos intra-sujeto entran dentro del término de EC, tal como pretende usarse en este trabajo.

\section{Clasificación de los estudios de casos}

Existen diversos modos de agrupamiento posibles al analizar los EC (ver Starman, 2013; para un panorama más amplio en este sentido). No es el objetivo de este artículo dar cuenta de todos ellos sino destacar dos clasificaciones que son relevantes: las vinculadas con factores temporales y las diferentes formas de vinculación entre el dato obtenido y el marco teórico.

Teniendo en cuenta factores temporales pueden considerarse tres tipos de estudio (Thomas, 2011): retrospectivos, trasversales (snapshot studies) y diacrónicos.

Los estudios retrospectivos plantean el estudio de un hecho pasado y sus implicancias actuales. Por ejemplo, Wanlass, Moreno, y Thomson (2005) estudiaron retrospectivamente un proceso de terapia grupal hospitalaria para trastornos alimentarios, recolectando información de terapeutas y pacientes sobre lo que habían considerado importante, útil y problemático del dispositivo terapéutico. Esto les permitió comprender las implicancias subjetivas que determinadas situaciones propias de la terapia y también del contexto tuvieron en los diferentes agentes.

Los estudios trasversales examinan un período específico de tiempo y buscan dejar constancia de la globalidad en contexto del fenómeno estudiado tal como sucedió. Un ejemplo de este tipo de estudios es el de Crepeau y Garren (2011) que realizan un EC en el que analizan trasversalmente el vínculo terapéutico entre un paciente y su terapeuta utilizando entrevistas y narraciones con el objetivo de comprender en profundidad esa relación. Aquí, el caso está definido como el vínculo entre terapeuta y paciente durante un período acotado.

Finalmente, los estudios diacrónicos intentan dar cuenta de los cambios a lo largo del tiempo y son equiparables a los estudios longitudinales Este último tipo de estudio es especialmente frecuente en psicoterapia donde se sigue un caso a lo largo de un proceso terapéutico observando los cambios que se producen en un sujeto o grupo de sujetos como resultado de un proceso terapéutico.

Si tenemos en cuenta la relación entre la recolección de datos y la teoría, Starman sugiere seguir la clasificación de George y Bennett (2005, citado por Starman, 2013). Los autores dividen los estudios de caso en: ateóricos, configurados por la teoría, heurísticos, de testeo de hipótesis, prueba de plausibilidad, y de construcción por bloques. 
Los estudios ateóricos, pretenden ser descriptivos y no contribuir directamente con el desarrollo conceptual, sino agotarse en la particularidad del fenómeno. Por ejemplo, estudios en los que se describe la experiencia de una persona en una situación determinada. Eso no inhibe obviamente al lector de formular sus propias teorías a trabajos científicos posteriores para analizar a la luz de una teoría determinada el caso en cuestión.

Los estudios configurados por la teoría son aquellos que utilizan abiertamente un marco teórico para explicar los fenómenos. El investigador, desde su teoría, configura la recolección de datos y los modelos explicativos.

Los estudios heurísticos buscan identificar fenómenos nuevos e inesperados para la teoría, a través del seguimiento de casos que se salen de lo común en algún sentido.

Los estudios de testeo de hipótesis buscan evaluar la validez de teorías. Si la hipótesis está planteada en términos apropiados, la presencia de resultados en un único caso ya resulta extremadamente significativa.

Los estudios de plausibilidad buscan verificar que futuras investigaciones sean factibles, por ejemplo Morris et al. (2010) recientemente realizaron un Estudio de Caso múltiple para evaluar la plausibilidad del uso de una aplicación para teléfonos celulares con el fin de favorecer la regulación emocional. Con este objetivo, entrenaron a 5 personas en el uso de una aplicación diseñada a tal fin y solicitaron que narraran sus experiencias respecto a la técnica. Los autores concluyen que el uso de la aplicación es posible y recomiendan realizar estudios de mayor magnitud muestral.

Finalmente, los estudios de construcción por bloques, son aquellos que hacen énfasis en aspectos muy puntuales de un fenómeno con la intención de contribuir luego a teorías más generales. Por ejemplo, el estudio del uso de una técnica terapéutica que luego formará parte de un paquete de intervenciones.

\section{Tipos de metodología en estudios de caso en psicoterapia}

Centrados en la investigación en psicoterapia, Iwakabe y Gazzola (2009) consideran tres tipos de EC: los clínicos, los experimentales y los estudios pragmáticos.

\section{Estudios de casos clínicos}

Consisten en narraciones por parte del terapeuta de lo sucedido a lo largo de un caso. Stiles les adjudica un valor científico equiparable a los estudios estadísticos, aunque con una utilidad claramente diferente y sin desconocer sus limitaciones. Para el autor la utilidad de los casos clínicos está en comparar proposiciones teóricas con observaciones en contexto (Stiles, 2005). 
Stiles propone el término de "triangulación" para referirse al funcionamiento de EC (Stiles, 2005). Desde un punto de vista geométrico, triangular implica fijar un punto en el espacio a partir de otras dos ubicaciones. Los resultados de los EC se situarían en un punto de entrecruzamiento entre las proposiciones teóricas y las observaciones en la búsqueda de confrontar estas últimas con el uso clínico de las teorías. Esto permite contrastar, refutar o modificar las teorías en función de los resultados.

La fragilidad metodológica de este tipo de estudios reside en muchos casos en que un único (e interesado) observador da cuenta de las características del paciente, de las intervenciones y de los resultados. Así mismo, muchos EC Clínicos, no siguen las guías metodológicas para estudios cualitativos actualmente disponible (Iwakabe \& Gazzola, 2009).

Dattilio (2006) en un intento de dotar de rigurosidad a este tipo de EC sugiere que los reportes de casos clínicos en terapia familiar deberían incluir siempre una serie de pasos que se explican en la Tabla 1.

\section{Tabla 1.}

Características necesarias de un estudio de caso (Dattilio, 2006, p. 211).

\begin{tabular}{l}
\hline 1. Observaciones, medidas y registros del comportamiento del paciente pre-tratamiento \\
\hline 2. Inferencias (sobre el paciente en su contexto biopsicosocial). \\
\hline 3. El tratamiento propuesto y las expectativas de logros. \\
\hline 4. Observaciones sobre el tratamiento que efectivamente se aplicó. \\
\hline $\begin{array}{l}\text { 5. Observaciones, medidas y registros sobre los cambios en los miembros de la familia } \\
\text { bajo tratamiento. } \\
\text { 6. Inferencias realizadas de estas observaciones. (Incluyendo la efectividad de los trata- } \\
\text { mientos aplicados) }\end{array}$
\end{tabular}

7. Una evaluación general del reporte del caso en función de las revisiones de pares.

\section{Estudio de caso experimental}

Refiere a un tipo de estudio también denominado en la literatura como estudio intrasujeto o experimento de $\mathrm{N}=1$. Stiles (2005) lo agrupa dentro de lo que él denomina "Prueba estadística de Hipótesis" y lo separa conceptualmente de los "Estudios de Caso" ya que su foco de abordaje es netamente cuantitativo.

Los estudios de caso experimentales son según Iwakabe y Gazzola de un método "riguroso para testear hipótesis sobre los efectos de un tratamiento y pueden considerarse una alternativa a estudios de gran escala sobre resultados" (Iwakabe \& Gazzola, 2009, p. 602). Este método, utilizado en general por las terapias conductuales y cognitivo conductuales, tiene el objetivo de registrar 
cambios específicos observables en un paciente que puedan ser atribuibles a una intervención. Se vale de técnicas cuantitativas para mesurar el estado del paciente en diferentes momentos del tratamiento.

Sin que esto implique una crítica sobre su utilidad como se mencionó en la introducción, si bien indudablemente trabajan con un "caso", sería forzado considerar este tipo de estudios dentro de los de EC tal como se los ha definido anteriormente.

\section{Estudios de casos pragmáticos en psicoterapia}

Los estudios pragmáticos surgen dentro del campo de la psicoterapia como forma de documentar un proceso terapéutico en profundidad. Intenta en este sentido dar cuenta de cómo cada terapeuta pone en juego sus creencias teóricas y las utiliza en el marco de su trabajo con cada paciente en particular. El producto de este tipo de EC busca que el lector comprenda lo que sucedió en un proceso terapéutico puntual sin resignar rigurosidad científica. Para esto, estandariza el proceso de análisis y recolección de datos; utiliza técnicas mixtas y, exige que todo el material sea analizado por un equipo de investigación y no de forma individual.

Según McLeod y Cooper (2011, p. 48) los estudios pragmáticos consisten en cuatro elementos principales:

1. El manejo cuidadoso de los aspectos éticos vinculados al consentimiento informado y a la confidencialidad.

2. La construcción de un registro de datos rico, que incluya información sobre el proceso y los resultados y que utilice un sistema mixto de datos cuantitativos y cualitativos (ver Tabla 2).

3. Un análisis de datos realizado en equipo en el cual se consideren tanto las interpretaciones convergentes como las divergentes.

4. La presentación de los datos de forma tal que permitan al lector monitorear el proceso de recolección de datos, el análisis y evaluar de forma independiente la credibilidad de las conclusiones. 
Tabla 2.

Puntos que componen un Registro de datos rico (McLeod \& Cooper, 2011).

\begin{tabular}{l}
\hline 1. Información del paciente previa a la terapia \\
\hline 2. Como mínimo una medición estandarizada de resultados administrada sesión \\
por sesión. \\
\hline 3. Como mínimo una medición de la calidad de la alianza terapéutica. \\
\hline 4. Información obtenida regularmente de paciente y del terapeuta sobre el pro- \\
ceso de la terapia \\
\hline 5. Las notas del terapeuta. \\
6. Copias de todo documento generado durante la terapia como por ejemplo \\
dibujos, diagramas, autoregistros, etc. \\
\hline 7. Grabación de las sesiones de psicoterapia. \\
8. Epicrisis del terapeuta. \\
\hline 9.Entrevistas de seguimiento.
\end{tabular}

Para sortear las limitaciones que se generan inevitablemente cuando un terapeuta, de forma solitaria, recolecta y analiza los datos; se utiliza un equipo de investigadores que analizan de forma independiente el caso y luego comparan sus hallazgos. Esto evita dudas o críticas respecto a los sesgos propios del terapeuta respecto de su trabajo. Los equipos se organizan de formas variadas siguiendo el principio básico de que las conclusiones pueden ser generadas por un proceso de diálogo en el cual las interpretaciones contrapuestas son evaluadas en términos de su capacidad de ajuste con los datos. La forma usual en que opera el equipo es la siguiente: el investigador principal toma la responsabilidad de unir todos los datos del caso en un "libro del caso". Copias de este libro son entregadas a cada miembro del equipo. Éste se reúne para acordar los procedimientos a realizar y luego cada investigador realiza un trabajo de interpretación de forma independiente. Una vez realizado este proceso, los investigadores comienzan una serie de reuniones en las que analizan acuerdos y discrepancias hasta arribar a un consenso.

Según sus creadores, esta forma de EC pueden permitir responder diferentes preguntas respecto al proceso terapéutico: respecto a los resultados ¿cuán efectiva fue la terapia en este caso?; respecto a la teoría ¿cómo pueden interpretare teóricamente los datos del caso en particular?; preguntas pragmáticas ¿qué estrategias y métodos usó el terapeuta en este caso? ¿cómo adaptó las técnicas para cumplir con las particularidades del paciente? y preguntas experienciales y narrativas ¿cómo fue la experiencia de ser paciente o terapeuta en este caso? ¿cuál es la historia de lo sucedido desde la perspectiva de cada uno? (McLeod \& Cooper, 2011, p. 54). 


\section{Ventajas de los estudios de casos}

Diversos autores han mostrado su preferencia y señalado las ventajas de los EC en psicoterapia (Edwards, Dattilio, \& Bromley, 2004; Dattilio, 2006; Stiles, 2006; Starman, 2013).

Stiles (2006) destaca que los clínicos siempre han manifestado su disconformidad con los estudios estadísticos en psicoterapia, pues estos están descontextualizados y son difíciles de aplicar en la práctica y considera que los EC pueden ayudar a acortar esta brecha. De forma inversa, también destaca que los Estudios de Caso pueden permitir a los clínicos incursionar en las investigaciones documentando adecuadamente sus casos, ya que son estos quienes están en una posición privilegiada para realizar observaciones ecológicas del objeto de estudio. Esto permitiría volver bidireccional el puente entre investigación y práctica clínica.

Starman (2013) utiliza el término de "validez conceptual" de la información recabada. Esto se refiere a la relación entre el fenómeno que quiere investigarse y la información obtenida: los estudios cuantitativos, mediante técnicas como los cuestionarios o las entrevistas estructuradas pueden facilitar la recolección de un flujo de datos extremadamente abundante, pero cuando se trata de conceptos complejos, como por ejemplo, "felicidad" o "frustración", es posible que solo mediante un estudio en profundidad que tenga en cuenta variables contextuales pueda capturarse la complejidad del fenómeno. En otras palabras, aunque la operacionalización de variables de forma que puedan cuantificarse es necesaria, toda operacionalización recorta los fenómenos, en algunos casos, al punto de permitir captar sólo una parte de lo que se pretende observar.

Otra ventaja de los Estudios de Caso es que, al trabajar con el caso en su contexto, se hace posible identificar variables nuevas y formular hipótesis complementarias. Mientras que los métodos más rígidos plantean modelos donde se va de la hipótesis a su prueba o refutación, el estudio de caso permite un tipo de diseño en el que la hipótesis y las variables pueden redefinirse a partir de la información obtenida en el campo (Rubaie, 2002). Con justicia puede argumentarse que este tipo de ventajas pueden extenderse en general a las metodologías cualitativas, al menos si se utiliza un diseño de investigación interactivo como el sugerido por Maxwell (1995) para superar la lógica lineal importada de las metodologías cuantitativas.

Starman (2013) considera también que los estudios de caso son especialmente útiles para explorar mecanismos causales. Permiten evaluar de forma holística un gran número de variables observando cualquier aspecto inesperado en el proceso. 
En estudios cuantitativos, aún con el uso de modelos causales complejos, se está dentro de la lógica de la probabilidad, sin la observación directa del fenómeno y sus devenires.

\section{Críticas a los estudios de casos}

\section{Falta de rigurosidad}

Se trata de una crítica frecuente y probablemente justificada en algunos casos. Defensores de este tipo de investigaciones manifiestan su preocupación respecto a la cantidad de trabajos que bajo el rotulo de EC se autorizan a utilizar metodología que carecen de rigor científico (Yin, 2003; Kyburz-Graber, 2004).

Los EC pueden ser extremadamente rigurosos, pero deben buscarse criterios de evaluación propios de los EC para juzgar la calidad metodológica y no intentar evaluar las investigaciones con categorías extrapoladas de lógicas cuantitativas. Así, podría hablarse de una forma de precisión propia de los EC, con sus propias reglas, en lo que se ha denominado "una subjetividad disciplinada" (Thomas, 2011).

Otro aspecto que suele achacarse como una limitación es el tamaño muestral. Esto es en sí mismo un error conceptual ya que la ciencia no requiere necesariamente grandes números para probar una teoría y menos aún para permitirse formularla. Si se acepta el principio de falsación popperiano (Flyvbjerg, 2006), en el cual los experimentos buscan refutar hipótesis y no verificarlas, podemos fácilmente comprender, que un solo caso posee valor y validez científica. Para poner un ejemplo algo burdo, bastó la explosión de una sola bomba atómica para probar el poder destructivo de las reacciones nucleares en cadena, o un solo viaje al espacio para probar que esto era posible. La utilidad y rigurosidad, no deben buscarse en la cantidad de la muestra sino en el diseño experimental. Existen obviamente preguntas de investigación que necesariamente requieren de grandes muestras y la replicación de un estudio arroja siempre mayores niveles de certeza.

\section{Falta de posibilidades de generalización}

Se ha escrito mucho sobre las posibilidades de generalización de los estudios de casos (Evers y Wu, 2006). Staker considera que “...el estudio de casos es una base pobre para poder generalizar (Staker, 2005, p. 19). Solo se estudia un caso, o unos pocos casos, pero se estudian en profundidad" y más adelante agrega: "El cometido real del estudio de casos es la particularización, no la generalización. Se toma un caso particular y se llega a conocerlo bien, y no principalmente para ver en qué 
se diferencia de los otros, sino para ver qué es, qué hace". Para Staker, entonces, si bien la generalización es siempre en alguna medida posible, no es un objetivo central. Evers y Wu (2006) señalan en este mismo sentido que un caso posee más estructuración y sustancia de lo que puede suponerse al traer consigo una cultura, un lenguaje y un ambiente que son comunes a otros casos. Es por eso que un estudio de caso realizado apropiadamente puede ser altamente representativo.

Yin (2003) realiza consideraciones interesantes sobre este punto. En principio destaca que aún en experimentación cuantitativa "pura" la generalización difícilmente sea válida a partir de un experimento, sino que es la sucesión de múltiples experimentos en ambientes diversos la que permite inferir. Por otro lado, diferencia entre dos tipos de generalización, la estadística y la analítica. En la primera se busca enumerar frecuencias de aparición, correlaciones y causalidades estadísticas (principalmente a través de regresiones) y en la segunda se busca expandir y generalizar teorías. Es sobre este tipo de generalización sobre la que se opera en este caso. Según Yin "los estudios de casos, como los experimentos, son generalizables a proposiciones teóricas y no a poblaciones o universos" (Yin, 2003, p. 10).

\section{Sesgos del investigador}

Stiles señala dos críticas que se aplican específicamente al campo de la investigación en psicoterapia (Stiles, 2005): por un lado la selección de la muestra puede ser vista como tendenciosa ya que es realizada de forma arbitraria por la persona que tiene una hipótesis previa respecto a lo que pretende encontrar en el campo. Por el otro, la forma de valorar progresos o retrocesos, en ausencia de medidas estandarizadas y de un lenguaje unificado puede conducir a numerosas imprecisiones. En este sentido, varios autores ya mencionados (McLeod \& Cooper, 2011; Dattilio, 2006) sugieren que el proceso sea revisado por pares o directamente, realizado por varios observadores.

\section{El rol del estudio de casos en la investigación en psicoterapia}

A lo largo del tiempo, y aún hoy, existe en el ámbito académico una dicotomía entre los investigadores que adhieren a una metodología netamente cuantitativa y aquellos que optan por estudios cualitativos. Como se ha dejado plasmado en este artículo, la realidad está lejos de estas simplificaciones y la pregunta adecuada parecería ser ¿para qué objetivo de investigación es pertinente qué metodología?

Edwards, Dattilio y Bromley (2004) proponen un modelo que se reproduce con adaptaciones en la Figura 1. En él se da cuenta del inter-juego deseable entre los diferentes eslabones que componen el desarrollo teórico en psicoterapia. 
En este modelo, el EC tiene un papel específico en el proceso de elaboración de teorías sobre la psicoterapia. Por un lado, constituye en ocasiones el primer paso de investigación cuando a partir de observaciones clínicas y del diálogo entre colegas se generan hipótesis (vínculo entre A y B en la Figura 1). Por otro lado, constituye la primera forma de aplicación práctica de hipótesis teóricas derivadas tanto de las ciencias básicas (como la neurociencia o la psicología cognitiva) como de la propia clínica psicológica (vínculo entre E y B en la Figura 1). Finalmente, el EC tiene un particular interjuego con los modelos de tratamiento clínico (vínculo B y D) que a su vez da lugar a la posibilidad de estudios estandarizados de gran escala (vínculo entre D y F). Este vínculo es el siguiente: por un lado, a partir de los EC se ponen a prueba la eficacia de determinados procedimientos y de los resultados de determinados paquetes de intervenciones que luego se cristalizan en un manual que puede ser testeado mediante ECR; por el otro, los EC permiten el análisis en profundidad de casos (pacientes, grupos de pacientes) en lo que se utilizó un protocolo estandarizado y hace visibles segmentos específicos del tratamiento, variables y condicionamientos que posibilitan realizar hallazgos que luego se plasman en adaptaciones y mejoras de los protocolos.

Cabe destacar que en el modelo, el vínculo entre la metodología y los estándares científicos (H) y los EC (B) señala también cómo los EC deben cumplir con una metodología rigurosa, aceptada por la comunidad científica.

Modelos como el reseñado, muestran la importancia del uso estratégico de diferentes formas de investigación de acuerdo por un lado al estadio del desarrollo de la teoría y por otro a las preguntas que se pretenden responder.

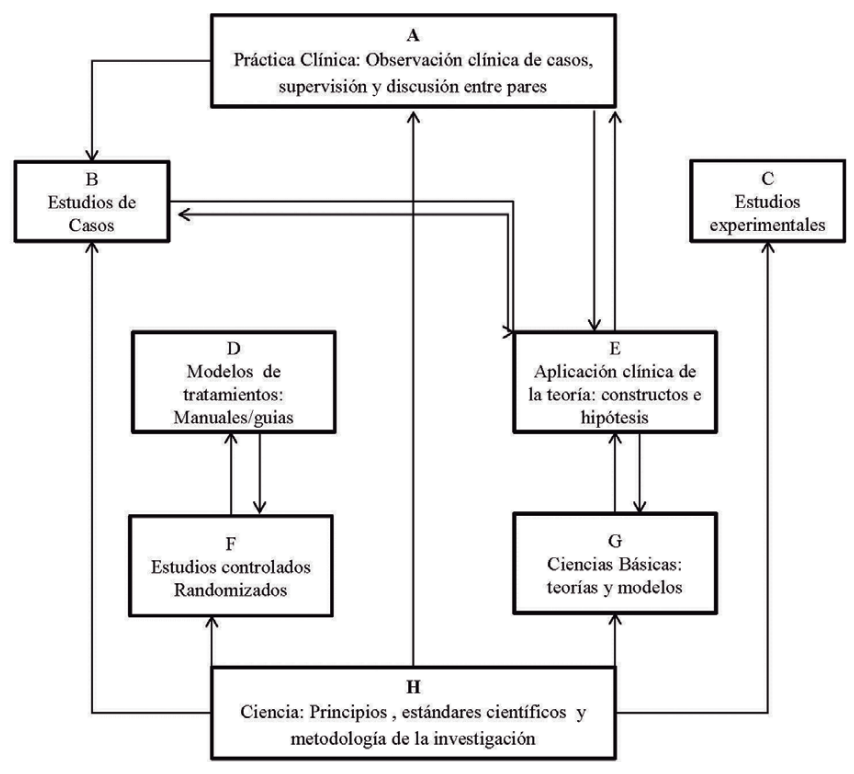

Figura 1. El rol de la investigación clínica en el desarrollo teórico en psicoterapia (Adaptado de Edwards, Dattilio \& Bromley, 2004). 


\section{Discusión}

Aunque diversas investigaciones se autodenominan EC, nos referimos a este tipo de trabajo cuando buscamos un conocimiento profundo y sistemático sobre un fenómeno particular dentro de su contexto.

Resultan necesarias guías y consensos sobre los procedimientos que componen este tipo de investigaciones de forma tal de garantizar una rigurosidad que permita a la comunidad científica confiar en los resultados obtenidos. Éstas debe comprender las características propias de los EC y no extrapolando criterios de validación propios de los métodos cuantitativos.

Resultan prometedores los trabajos contemporáneos que buscan sistematizar tanto la recolección de datos como el análisis de los mismos respetando el espíritu fenomenológico de los EC y tomando en cuenta las críticas más frecuentes que este tipo de trabajos recibe. En este sentido, la revisión por pares y el trabajo en equipo son especialmente útiles para evitar posibles sesgos al tiempo que estandarizaciones sobre el modo en que los datos son recolectados garantizan la calidad de los mismos.

La loable búsqueda de mayores estándares científicos para la psicoterapia, no debe impedirnos ver el rol que los EC ocupan en el desarrollo de la misma. El objetivo debe ser coordinar de forma eficiente los diferentes tipos de diseños de investigación disponibles teniendo en cuenta para ello, las ventajas que cada uno puede aportar dependiendo del tipo de pregunta que espera responderse. 


\section{Referencias}

Dattilio, F. M. (2006). Case-Based Research in Family Therapy. Australian and New Zealand Journal of Family Therapy, 27(4).

Edwards, D. J. A., Dattilio, F. M., \& Bromley, D. B. (2004). Developing evidencebased practice: the role of case-based research. Professional Psychology: Research and Practice, 35, 589-597.

Evers, C. W., \& Wu, E. H. (2006). On Generalising from Single Case Studies: Epistemological Reflections. Journal of Philosophy of Education, 40(4), 511-526.

Flyvbjerg, B. (2006). Five Misunderstandings About Case-Study Research. Qualitative Inquiry, 12(2), 219-245.

Iwakabe, S., \& Gazzola, N. (2009). From single-case studies to practice-based knowledge: aggregating and synthesizing case studies. Psychotherapy research: journal of the Society for Psychotherapy Research, 19(4-5), 601-611.

Kazdin, A. E., \& Blase, S. L. (2011). Rebooting Psychotherapy Research and Practice to Reduce the Burden of Mental Illness. Perspectives on Psychological Science, 6(1), 21-37.

Kyburz-Graber, R. (2004). Does case-study methodology lack rigour? The need fort quality criteria for sound case-study research, as illustrate a recent case in secondary and higher education. Environmental Education Research, 10(1), $53-65$.

Luck, L., Jackson, D., \& Usher, K. (2006). Case study: a bridge across the paradigms. Nursing inquiry, 13(2), 103-109.

McLeod, J. \& Cooper, M. (2011). A protocol for systematic case study research in pluralistic counseling and psychotherapy. Counseling Psychology Review, 26(4), 47-58.

Maxwell, J. (1996) Qualitative research design. An interactive Approach. Sage publications, 1-13.

Morris, M. E., Kathawala, Q., Leen, T. K., Gorenstein, E. E., Guilak, F., Labhard, M., \& Deleeuw, W. (2010). Mobile Therapy: Case Study Evaluations of a Cell Phone Application for Emotional Self-Awareness. Journal of Medical Internet Research, 12, e10. 
Rubaie, T. A. (2002). The rehabilitation of the case-study method. European Journal of Psychotherapy \& Counseling, 5(1), 31-47.

Stake, R. E. (2000). Case Studies. En N. K. Denzin \& Y. S. Lincoln (Eds.), Handbook of Qualitative Research. Thousand Oaks, CA: Sage Publications.

Staker, R. (2005). Investigación con estudios de casos. En Investigación con estudios de casos. Madrid: Morata, 15-24.

Starman, A. B. (2013). Adrijana Biba Starman The case study as a type of qualitative research. Journal of Contemporary Educational Studies, 1, 28-43.

Stiles,W. B. (2005). Case studies. In J. C. Norcross, L. E. Beutler, \& R. F. Levant (Eds.), Evidence-based practices in mental health: Debate and dialogue on the fundamental questions. Washington, DC: American Psychological Association, 57-64.

Tight, M. (2010). The curious case of case study: a viewpoint. International Journal of Social Research Methodology, 13(4), 329-339.

Thomas, G. (2011). A typology for the case study in social science following a review of definition discourse and structure. Qualitative Inquiry, 17(6), 511-521.

Wanlass, J., Moreno, J. K., \& Thomson, H. M. (2005). Group Therapy for Eating Disorders: A Retrospective Case Studty. The Journal for Specialist in Group Work, 30(1), 47-66.

Yin, R. K. (2003). Case study Research: Design and Methods. (3ra ed.). Thousand Oaks, California: Sage Publications, INC. 